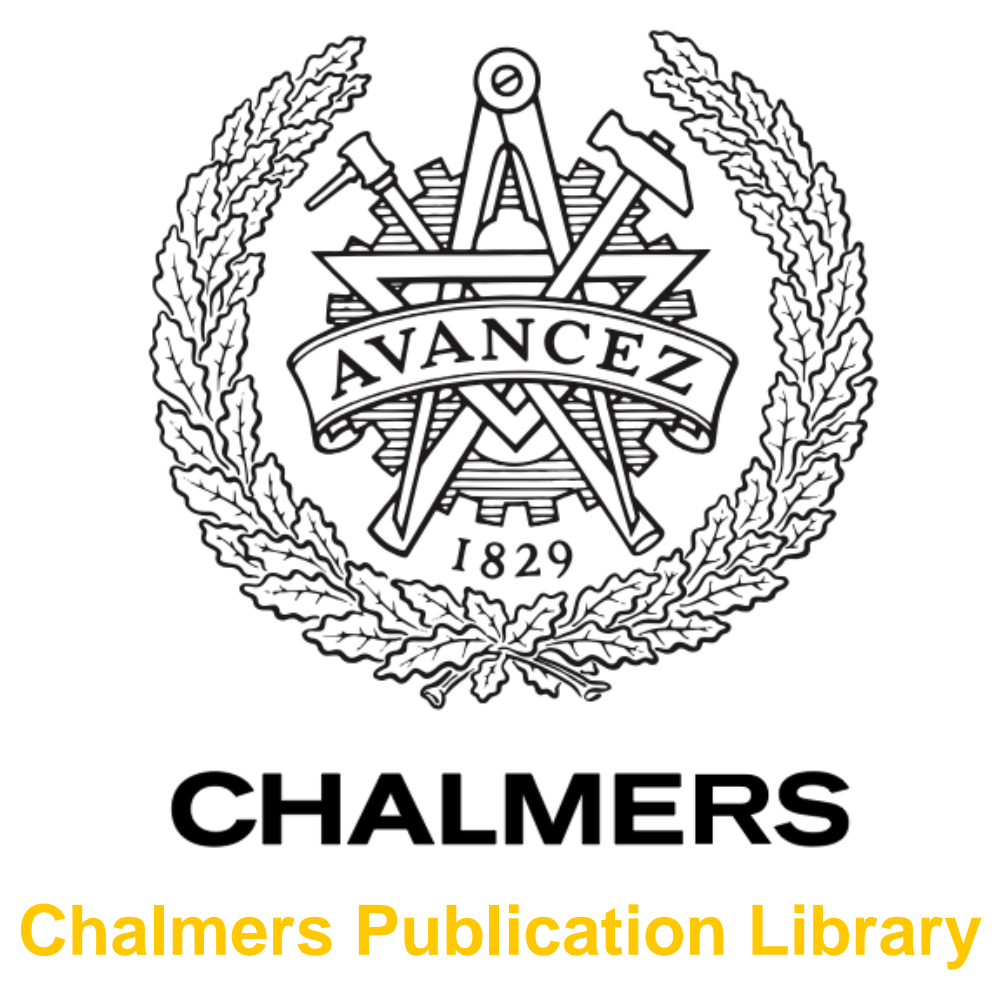

\title{
Simultaneous Information and Power Transfer under a Non-Linear RF Energy Harvesting Model
}

This document has been downloaded from Chalmers Publication Library (CPL). It is the author's version of a work that was accepted for publication in:

IEEE International Conference on Communications Workshops (ICC) (2017)

Citation for the published paper:

Xu, X. ; Ozcelikkale, A. ; McKelvey, T. et al. (2017) "Simultaneous Information and Power Transfer under a Non-Linear RF Energy Harvesting Model". IEEE International Conference on Communications Workshops (ICC) (2017)

Downloaded from: http://publications.lib.chalmers.se/publication/248512

Notice: Changes introduced as a result of publishing processes such as copy-editing and formatting may not be reflected in this document. For a definitive version of this work, please refer to the published source. Please note that access to the published version might require a subscription. 


\title{
Simultaneous Information and Power Transfer under a Non-Linear RF Energy Harvesting Model
}

\author{
Xiaowei Xu, Ayça Özçelikkale, Tomas McKelvey, Mats Viberg
}

\begin{abstract}
In the design of simultaneous wireless information and power transfer (SWIPT) systems, it has been typically assumed that energy conversion efficiency is independent from the level of the input power at the energy receiver. On the other hand, in practice the energy conversion efficiency exhibits a nonlinear behavior and highly depends on the input power. This leads to a discrepancy between the practical energy harvesting (EH) hardware available and the resource allocation designs made for the SWIPT systems. This work is concerned with this issue. In particular, we propose a practical quadratic model for the power conversion efficiency in EH circuitry. Comparisons with the constant efficiency models used in conventional SWIPT system design as well as another non-linear model proposed in the literature are made. With its convexity properties together with the good match it provides for the measurement data from practical EH circuitry, the proposed model is shown to be a promising alternative to the existing $\mathrm{EH}$ approaches. Using the proposed model, the problem of resource allocation for a multiuser Orthogonal Frequency-Division Multiple Access (OFDMA) system is investigated. The performance improvement due to the usage of the proposed non-linear model is illustrated.
\end{abstract}

\section{INTRODUCTION}

Wireless power transfer through RF signals has recently received considerable attention as an attractive alternative for powering up wireless sensor networks. On the other hand, RF signals are already widely used for transmitting information, namely wireless information transfer. Accordingly, the promising idea of integrating these two tasks, referred as simultaneous wireless information and power transfer (SWIPT), has recently emerged [1-3]. In SWIPT systems, RF signals are used to transmit information to information receivers and power to energy receivers, simultaneously. SWIPT system design has been studied under various scenarios [1-7], including broadcast channels [3], relay channels [4], interference channels [5], [6] and orthogonal frequency division multiple access (OFDMA) systems [7].

Works on SWIPT system design in communication community typically adopt energy harvesting models that assume ideal hardware. In this ideal hardware models, the average power harvested at the end of EH circuity is considered to be a linear function of the average input power to the device. Practical issues, such as the non-linear dependency of the energy harvested on the input power or the dependency on the

$\mathrm{X} . \mathrm{Xu}$ is in Communication Eng. and A. Özçelikkale, T. McKelvey and M. Viberg are with Dep. of Signals and Systems, Chalmers University of Technology, Gothenburg, Sweden e-mails: xiaowei@student.chalmers.se, \{ayca.ozcelikkale, tomas.mckelvey, mats.viberg\}@chalmers.se. A Ozçelikkale acknowledges the funding support from the European Union Horizon 2020 research and innovation programme under grant agreement No 654123 . input wave-form, is typically overlooked. On the other hand, these non-linear characteristics are of central importance in the design of practical EH circuitry [8-11].

Works that incorporate these practical hardware considerations in wireless power transfer system design have only recently started to emerge. Refs. [12-14] study waveform design for power transfer under practical models and show the superior performance of multi-sine waveforms compared to traditional communication waveforms. A non-linear model for power conversion efficiency in EH circuitry is proposed [15] and significant improvements on the system performance compared to the performance of the designs utilizing conventional linear models are illustrated [15-18]. Nevertheless, from a system design point of view, the trade-offs between the ease of analysis and the performance degradation due to the usage of linear or non-linear models is not completely understood at the moment. Here, we address this challenge by focusing on the non-linear power conversion efficiency and proposing a practical model that provides a reasonable trade-off between tractability and accuracy.

In particular, the works that focus on hardware design for RF energy harvesting [8-11] reveal that the efficiency of the conversion from the power that the ER receives and the power that can be stored by the ER is highly dependent on the input power. More specifically, the power conversion efficiency first increases as the input power increases until a certain value and then decreases as the input power further increases [8-11]. Hence the power conversion efficiency is not constant and the relationship between the output power that can be stored and the input power is not linear.

In contrast to the above, a linear model is used in conventional SWIPT system design. An important exception is the work of [15] which proposes a non-linear model. Utilizing this model, Refs. [15-18] show that usage of linear model may lead to significant performance loss. Thus having a suitable non-linear power conversion model is crucial for obtaining the optimum performance for the SWIPT systems.

In this work, we further explore this point and propose a quadratic function as an alternative non-linear model for power conversion efficiency. We compare our proposed model with the traditional linear model and the model of [15]. Similar to the logistic function model of [15], the proposed quadratic model provides a good match with the measurement data. In contrast to the model of [15], the proposed model is a concave function of the input power, which is a desirable property from the point of view of tractability. These findings suggest that the proposed quadratic model provides a reasonable trade-off between tractability from the point of view of analysis, and 
the accuracy from the point of view of modeling practical EH circuitry. Using the proposed model, we consider a resource allocation problem for a SWIPT OFDMA system. Our results illustrate the performance improvement that can be obtained by using the proposed model compared to the traditional linear models. In particular, although designs based on a linear model can overestimate the power that should be used at the transmitter or may fail to actually satisfy the EH constraints, the proposed model provides a reasonable compromise between these two objectives.

The rest of the paper is organized as follows. In Sec. II, the proposed non-linear EH model is presented and the comparisons with the typical linear model in the literature and the non-linear of [15] are provided. The power allocation problem for SWIPT under OFDMA is presented in Sec. III. In Sec. IV, the performance of the designs based on the proposed nonlinear model is illustrated. The paper is concluded in Sec. V.

\section{Proposed Energy Harvesting Model}

Let $y_{E H}$ be the received signal at the energy receiver. The average power input to the energy receiver can be expressed as follows [3-7]:

$$
P_{\text {in }}=\mathbb{E}\left[\left\|y_{E H}\right\|^{2}\right] .
$$

Let the power that is extracted by the energy receiver be denoted by $P_{\text {out }}$. We consider the following two alternatives to model the conversion process between $P_{\text {in }}$ and $P_{\text {out }}$ :

- The linear model with a constant power conversion efficiency $\zeta \geq 0$ :

$$
P_{\text {out }}=\zeta P_{\text {in }}
$$

This is the model typically used in SWIPT system design [3-7].

- Power conversion efficiency $\zeta$ is dependent on $P_{i n}$ :

$$
P_{\text {out }}=f\left(P_{\text {in }}\right) P_{\text {in }},
$$

where (3) could be also expressed as

$$
P_{\text {out }}=g\left(P_{\text {in }}\right) \text {, }
$$

where $g($.$) is a non-linear function of P_{i n}$.

The literature on the hardware design for RF energy harvesting reveal that the efficiency of the conversion between $P_{\text {in }}$ and $P_{\text {out }}$ highly depends on $P_{\text {in }}$ [8-11]. Motivated by the measurement data in these works, we propose a $2^{\text {nd }}$ order polynomial model as the non-linear $g\left(P_{i n}\right)$ as follows:

$$
g\left(P_{i n}\right)=\alpha_{1} P_{i n}^{2}+\alpha_{2} P_{i n}+\alpha_{3},
$$

where $\alpha_{1} \in \mathbb{R}, \alpha_{2} \in \mathbb{R}, \alpha_{3} \in \mathbb{R}$ are the parameters of the model. This model provides a generic relationship between the input and output power at the energy receiver accounting for various non-linearities such as possible saturation effects or current leakage and limitations. The values of the parameters depend on the actual antenna design and circuitry used by the EH device and can be determined by data fitting tools. An illustration of the proposed model together with the linear models is provided in Fig. 1 using data from [9]. Here the

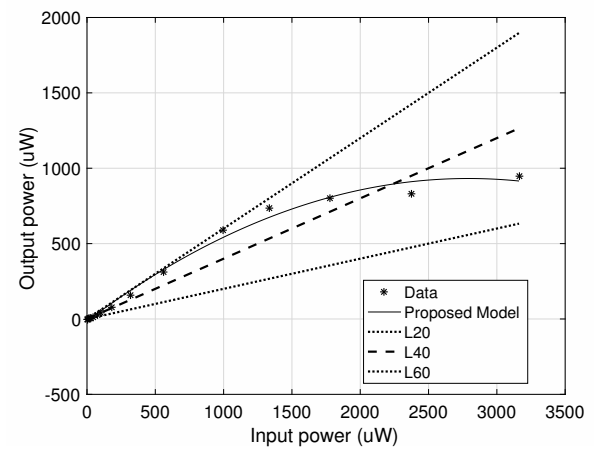

Fig. 1: Comparison between linear model and non-linear model. Measurement data is from [9].

data from [9] provides sample points where $P_{i n}$ and the corresponding $P_{\text {out }}$ is found using actual hardware designs [9]. The parameters $\alpha_{1}, \alpha_{2}, \alpha_{3} \in \mathbb{R}$ are determined using meansquare error based data fitting on the data from [9]. L20, L40 and L60 correspond to the linear model where $\zeta$ in (2) is chosen as $0.2,0.4$ and 0.6 , respectively. We observe that the proposed model provides a good match for the data at hand compared to the linear models.

RF energy harvesting hardware designs are typically optimized for a certain target input power range [8-11]. As a result, the power conversion efficiency typically first increases as the input power increases up until a certain input power value, and then it decreases as the input power further increases beyond this certain power value [8-11]. An illustration of this point is given in Fig. 1. We note that in order to match such a characteristics with a quadratic $g($.) function, one would typically have $\alpha_{1}<0$. Hence $g\left(P_{i n}\right)$ becomes a concave function of $P_{i n}$. Such a non-linear model may provide possible ease of design in SWIPT system resource allocation problems compared to other possible non-linear models: In a typical SWIPT resource allocation problem, the energy harvested is either maximized or a lower bound on the energy harvested is provided. Maximizing a concave function is consistent with a convex optimization formulation [19]. Similarly, lower bounding a concave function corresponds to a convex constraint [19]. Hence such a $g($.$) function enables optimization formulations$ that are convex which may be solved using the available numerical tools for convex optimization tools such as SeDuMi, SDPT3 and CVX [20-22] or may be easier to provide analytic insight through, for instance, due to sufficiency of KarushKuhn-Tucker (KKT) conditions under constraint qualification [19].

An important issue with any non-linear energy harvesting model that is found by data fitting is the range of input power values the model is valid. This issue stems from the fact that the measurement data is typically only available for a certain range of input power values. Although it is attractive to adopt a model that $P_{\text {out }}$ saturates after a certain $P_{\text {in }}$, it is not clear whether this will be the case with practical hardware; see for instance Fig. 7 of [9] where the conversion efficiency shows a trend of rapidly decreasing with increasing power. This behavior is related to the fact that hardware designs are typically optimized for a certain input power range. Consistent with this 


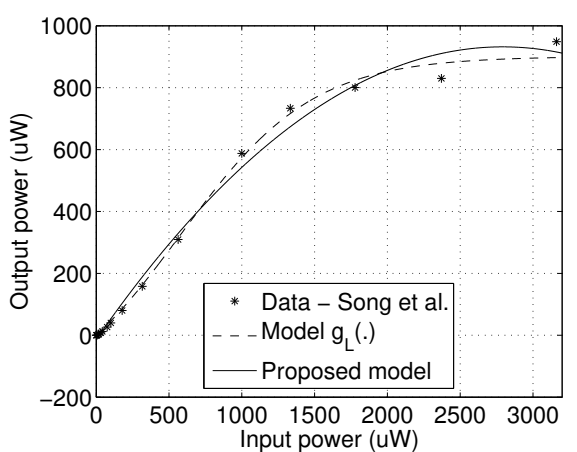

Fig. 2: Comparison between the model from [15] and the proposed model. Measurement data is from [9].

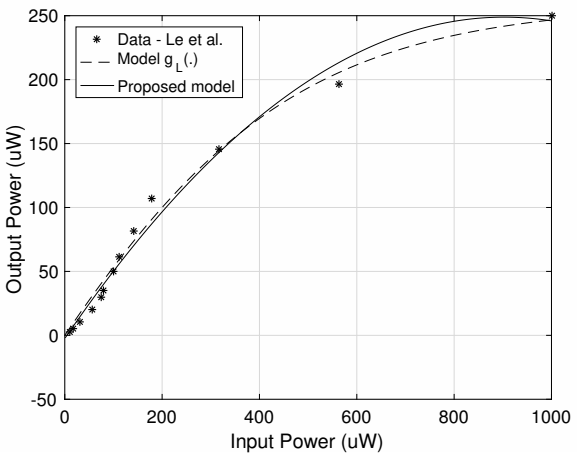

Fig. 3: Comparison between the model from [15] and the proposed model. Measurement data is from [10].

fact, we note that the proposed model is only valid under a certain $P_{i n}$ range which depends on the actual hardware used. We further discuss this point in Section III.

\section{A. Comparison with the non-linear model from [15]}

The possible non-linearity of the relationship between $P_{\text {out }}$ and $P_{i n}$ is typically overlooked in SWIPT system design. An important exception is Ref. [15] which proposes a non-linear model $g_{L}($.$) based on the logistic function:$

$$
\begin{gathered}
g_{L}\left(P_{i n}\right)=\frac{\bar{P}-\beta_{3} S}{1-S}, \\
\bar{P}=\frac{\beta_{3}}{1+\exp \left(-\beta_{1}\left(P_{i n}-\beta_{2}\right)\right)}, \\
S \triangleq \frac{1}{1+\exp \left(\beta_{1} \beta_{2}\right)},
\end{gathered}
$$

where $\beta_{1}, \beta_{2}, \beta_{3}$ are the parameters of the model. These parameters are again determined using data fitting tools. We now present a visual comparison of the fits provided by the proposed model and the model of [15]. The fit provided by these models using the data in [9] and [10] are shown in Fig. 2 and Fig. 3, respectively. At each case, the model parameters are found using the respective data set and mean-square error based data fitting. We observe that both the proposed model and the model of [15] provide a good match for the data in all cases.
TABLE I: Comparison of Goodness of Fit

\begin{tabular}{|l|l|l|l|}
\hline Data & Model & RSSE & $\mathrm{R}^{2}$ \\
\hline \multirow{2}{*}{ From [10] } & Model from [15] & 28.577 & 0.9889 \\
\cline { 2 - 4 } & Proposed Model & 32 & 0.9860 \\
\hline \multirow{2}{*}{ From [9] } & Model from [15] & 79.850 & 0.9969 \\
\cline { 2 - 4 } & Proposed Model & 125.857 & 0.9922 \\
\hline \multirow{2}{*}{ From [11] } & Model from [15] & 2804 & 0.9863 \\
\cline { 2 - 4 } & Proposed Model & 3007 & 0.9842 \\
\hline
\end{tabular}

We now investigate the goodness of the fit of these models using statistical measures; root-sum-of-squares error (RSSE) and the coefficient of determination, i.e. R-squared value $\left(\mathrm{R}^{2}\right)$ [23]. Let $n$ be the number of data points we have from the measurements. Let $P_{i}$ be the output power value at sample point $i$. Let $\hat{P}$ be the value predicted by the model. Let $\bar{P}$ be the average of the samples, i.e. $\bar{P}=\frac{1}{n} \sum_{i=1}^{n} P_{i}$. RSSE is defined as $R S S E=\sqrt{\sum_{i=1}^{n}\left(P_{i}-\hat{P}_{i}\right)^{2}}$. RSSE measures the total deviation of the response values from the values provided by the fit. A value closer to 0 suggests a better fit. $\mathrm{R}^{2}$ is defined as the ratio of the sum of squares of the regression $\left(\sigma_{R}^{2}\right)$ and the total sum of squares $\left(\sigma_{T}^{2}\right)$ where $\sigma_{R}^{2}$ is defined as $\sigma_{R}^{2}=$ $\sum_{i=1}^{n}\left(\hat{P}_{i}-\bar{P}\right)^{2}$ and $\sigma_{T}^{2}$ is defined as $\sigma_{T}^{2}=\sum_{i=1}^{n}\left(P_{i}-\frac{R}{P}\right)^{2}$. Hence $\mathrm{R}^{2}$ is defined as $R^{2}=\frac{\sigma_{R}^{2}}{\sigma_{T}^{2}}$ [23]. $\mathrm{R}^{2}$ takes values between 0 and 1 , with a value closer to 1 indicating that a greater proportion of variance in the data set is accounted for by the model.

The RSSE and $\mathrm{R}^{2}$ values are reported in Table I for measurement data sets in [9-11]. We observe that both models provide similar values for both measures. The values of RSSE for the proposed model are slightly larger than the model of [15]. For $\mathrm{R}^{2}$ the values for both models provides values that are very close. We note that $\mathrm{R}^{2}$ is a ratio and thus less dependent on the specific values of data. These results together with the visual inspection provided by Fig. 2 and Fig. 3 show that the proposed model provides a promising alternative in terms of representing the non-linear relationship between the input and output power.

The logistic function model of [15] in (6)-(8) is, in general, not a concave function of $P_{i n}$ [15]. Hence, for instance, maximizing the energy harvested under this model does not directly correspond to a convex optimization formulation. Hence the readily available numerical tools for convex optimization algorithms cannot be directly used and the applicability of some of the analytical tools, such as the sufficiency of the KKT conditions, should be further investigated. Although promising solutions with this non-convex model have been provided in [15], it is of interest to investigate viable alternatives. With its convexity properties together with the good match it provides for the practical measurement data, the proposed model in this work provides such a promising alternative.

\section{SWIPT IN OFDMA SYSTEMS}

We now illustrate the performance gain that could be obtained by utilizing the proposed non-linear model in SWIPT system design. We consider a single cell OFDMA downlink system where one base station serves $N$ mobile users [7]. 
The spectrum is divided into $N$ equal sub-channels. Each mobile is assigned one sub-channel. The effective channel gain at sub-channel $i$ after beamforming is denoted by $h_{i}>0$, $i=1, \ldots N$. The signal transmitted by the base station at sub-channel $i$ has the power of $Q_{i}, i=1, \ldots, N$. The noise in sub-channel $i$ is denoted by $\sigma_{i}^{2}$. Hence the receiver signalto-noise ratio for the sub-stream $i$ can be written as follows [7]

$$
S N R_{i}=\frac{\sigma_{i}^{2}+Q_{i} h_{i}}{\sigma_{i}^{2}} .
$$

The average input power for the receiver tuned to the substream $i$ can be expressed as follows

$$
P_{i n}^{i}=\sigma_{i}^{2}+Q_{i} h_{i}
$$

Each mobile user either extracts power or information from the received signal based on whether it acts as an information receiver or energy receiver. Let $S=\{1, \ldots, N\}$ denote the set of indices. Let $S_{E} \subseteq S$ and $S_{I} \subseteq S$, denote the set of indices of users that extracts information and energy, respectively where $S_{E} \cap S_{I}=\emptyset$. We consider the following problem that minimizes the transmission power under throughput and harvested energy constraints:

$$
\begin{array}{ll}
\min _{\left\{Q_{i}\right\}} & \sum_{i=1}^{N} Q_{i} \\
\text { s.t. } & \sum_{i \in S_{I}} \log \left(S N R_{i}\right) \geq C, \\
& \sum_{i \in S_{E}} g\left(P_{i n}^{i}\right) \geq \gamma \\
& P_{i n}^{i} \leq P_{i n}^{U}, \quad i \in S_{E} \\
& P_{i n}^{i} \geq P_{i n}^{L}, \quad i \in S_{E} \\
& Q_{i} \geq 0, \quad i=1, \ldots, N
\end{array}
$$

These type of resource allocation problems in OFDMA systems have been considered before under a linear energy harvesting model, see for instance [7]. We consider the problem under the proposed non-linear EH model.

Here (11d) is imposed due to the range of validity of the proposed EH model. We note that imposing (11d) is equivalent to considering a model that provides a saturation after $P_{i n}^{U}$ value (i.e. $P_{\text {out }}$ remains constant at the value $g\left(P_{i n}^{U}\right)$ for $P_{\text {in }} \geq$ $P_{i n}^{U}$ ). This is due to the fact that under a model with such a saturation property, there is no benefit of increasing the input power values $P_{i n}^{i}$ from the perspective of energy harvesting constraints whereas the objective function deteriorates with increasing $P_{i n}^{i}$, since this requires increasing $Q_{i}$. Hence under such a model optimal $Q_{i}$ are such that $P_{i n}^{i} \leq P_{i n}^{U}, i \in S_{E}$ are satisfied. Similarly, imposing (11e) is equivalent to considering an $\mathrm{EH}$ model that provides 0 output power for input power values smaller than $P_{i n}^{L}$.

We observe that $g($.$) is a concave function with \alpha_{1} \leq 0$. As discussed in Sec. II, models with $\alpha_{1} \leq 0$ provide good match for energy harvesting measurements in the literature. Hence we assume that $\alpha_{1} \leq 0$. Hence, (11c) bounds a concave function from below; which is equivalent to bounding a convex function from above. Hence, (11c) forms a convex constraint. Similarly, (11b) forms a convex constraint. Here (11e), (11d) and (11f) form linear, hence convex constraints. Hence, (11) constitutes a convex optimization problem. Therefore, given that Slater's condition is satisfied, KKT conditions are necessary and sufficient for optimality [19]. In particular, Lagrangian can be written as follows:

$$
\begin{aligned}
\mathcal{L}= & \sum_{i=1}^{N} Q_{i}+\lambda_{I}\left(C-\sum_{i \in S_{I}} \log \left(S N R_{i}\right)\right) \\
& +\lambda_{E}\left(\gamma-\sum_{i \in S_{E}} g\left(P_{i n}^{i}\right)\right)+\sum_{i \in S_{E}} \kappa_{i}^{U}\left(h_{i} Q_{i}+\sigma_{i}^{2}-P_{i n}^{U}\right) \\
& -\sum_{i \in S_{E}} \kappa_{i}^{L}\left(h_{i} Q_{i}+\sigma_{i}^{2}-P_{i n}^{L}\right)-\sum_{i=1}^{N} \mu_{i} Q_{i}
\end{aligned}
$$

where $\lambda_{I} \in \mathbb{R} \geq 0, \lambda_{E} \in \mathbb{R} \geq 0, \kappa_{i}^{U} \in \mathbb{R} \geq 0, \kappa_{i}^{L} \in \mathbb{R} \geq 0$, $\mu_{i} \in \mathbb{R} \geq 0$ are Lagrange multipliers. Hence together with the feasibility conditions, the KKT conditions can be expressed as follows:

$$
\begin{aligned}
& 1-\lambda_{I} \mathbb{1}_{i \in S_{I}} \frac{\bar{h}_{i}}{1+\bar{h}_{i} Q_{i}} \\
&-\lambda_{E} \mathbb{1}_{i \in S_{E}}\left(2 \alpha_{1} h_{i}^{2} Q_{i}+\alpha_{2} h_{i}\right) \\
&+\mathbb{1}_{i \in S_{E}} \kappa_{i}^{U} h_{i}-\mathbb{1}_{i \in S_{E}} \kappa_{i}^{L} h_{i}-\mu_{i}=0, \quad \forall i \\
& \lambda_{I}\left(C-\sum_{i \in S_{I}} \log \left(S N R_{i}\right)\right)=0, \\
& \lambda_{E}\left(\gamma-\sum_{i \in S_{E}} g\left(P_{i n}^{i}\right)\right)=0, \\
& \kappa_{i}^{U}\left(h_{i} Q_{i}+\sigma_{i}^{2}-P_{i n}^{U}\right)=0, \quad i \in S_{E} \\
& \kappa_{i}^{L}\left(h_{i} Q_{i}+\sigma_{i}^{2}-P_{i n}^{L}\right)=0, \quad i \in S_{E} \\
& \mu_{i} Q_{i}=0 \quad i=1, \ldots, N
\end{aligned}
$$

where $\mathbb{1}$ is the indicator function and $\bar{h}_{i}=h_{i} / \sigma_{i}^{2}$. For $i \in S_{I}$, the solution can be expressed as

$$
Q_{i}=\left(\lambda_{I}-\frac{1}{\bar{h}_{i}}\right)^{+}, \quad i \in S_{I}
$$

where $x^{+} \doteq \max (0, x)$. We observe that as the effective channel power increases, $Q_{i}$ increases. Due to the separability of the problem, this optimal solution for the information users is in the same general form with the optimal solution of the transmit power minimization problem under only throughput constraints. We now turn our attention to EH constraints. For $i \in S_{E}$, the solution can be expressed as

$$
Q_{i}=\frac{\left(\lambda_{E} \alpha_{2} h_{i}-1-\kappa_{i}^{U} h_{i}+\kappa_{i}^{L} h_{i}\right)^{+}}{\lambda_{E} 2\left|\alpha_{1}\right| h_{i}^{2}}, \quad i \in S_{E}
$$

where we have used $\alpha_{1} \leq 0$.

We note that due to the general trend of the RF energy harvesting measurement data, such as illustrated in Fig. 2, $g\left(P_{\text {in }}\right)=0$ will typically have one root that is close to 0 and one positive root with larger absolute value, hence $\alpha_{2}>0$. We also note that we typically have $g(0) \leq 0$, since it is likely that energy cannot be harvested until the input power exceeds a threshold, hence we have $\alpha_{3} \leq 0$. 
Let us now consider the case with equal effective channel signal-to-noise ratior on all sub-channels under these assumptions:

Lemma 3.1: Let $\alpha_{1} \leq 0, \alpha_{2}>0, \alpha_{3} \leq 0$. Let $h_{i}=h$, $\sigma_{i}^{2}=\sigma^{2}, \forall i$. If the following solution is feasible, then it forms an optimal solution:

$$
\begin{gathered}
Q_{i}=\frac{\sigma^{2}}{h}\left(e^{C / n_{I}}-1\right), \quad i \in S_{I} \\
Q_{i}=\frac{1}{h}\left(\bar{Q}-\sigma^{2}\right), \quad i \in S_{E} \\
\bar{Q}=\frac{1}{2 \alpha_{1}}\left(-\alpha_{2}-\sqrt{\alpha_{2}^{2}-4 \alpha_{1}\left(\alpha_{3}-\bar{\gamma} / n_{E}\right)}\right),
\end{gathered}
$$

where $n_{I}$ and $n_{E}$ is the cardinality of the set $S_{I}$ and $S_{E}$, respectively.

Proof: Let us consider an optimal solution $Q_{i}^{*}$. Let us have $\sum_{i \in S_{I}} Q_{i}^{*}=P_{I}$ and $\sum_{i \in S_{E}} Q_{i}^{*}=P_{E}$. We observe that under the given channel assumptions, (11b) is in the form of sum of identical concave functions. Hence it is Schur-concave and $Q_{i}=P_{I} / n_{I}$ maximizes (11b) under a constraint $\sum_{i \in S_{I}} Q_{i}=$ $P_{I}$ [24, Ch.3]. Hence there exists an optimal solution for (11) with $Q_{i}=P_{I} / n_{I}$ equal for $i \in S_{I}$ [24, Ch.3]. The same arguments hold for (11c) with the constraint $\sum_{i \in S_{E}} Q_{i}^{*}=P_{E}$. Such solutions are guaranteed to satisfy (11e)-(11d) due to the feasibility assumption. The expressions (20) and (22) follow from the uniformity of $Q_{i}$ over $S_{I}$ and $S_{E}$, respectively. For (22) we have used the smaller root of the following second order equation $\alpha_{1} P_{i n}^{2}+\alpha_{2} P_{i n}+\alpha_{3}-\gamma / n_{E}=0$, with $\alpha_{1} \leq 0$, $\alpha_{2}>0, \alpha_{3} \leq 0$

\section{NumericAl Results}

We now compare the performance of the designs made using the proposed model with the designs made using the typical linear EH model used in SWIPT system design. We consider the following power transmission model which relates the power at the transmitter $P_{T}$ to the received input power at the receiver [7], [25]:

$$
\frac{P_{T}}{P_{\text {in }}}=\frac{A_{t} A_{r}}{\lambda^{2} d^{2}}|Z|^{2},
$$

where $\lambda$ is the wavelength, $d$ is the transmission distance, $A_{t}$ and $A_{r}$ are the total apertures of the transmit and receive antenna arrays, respectively and $Z$ is a complex proper Gaussian random variable with non-zero mean. Let $N=4$, $f=v_{c} / \lambda=2.4 G H z, v_{c}=3 \times 10^{8} \mathrm{~m} / \mathrm{s}, A_{t}=0.1 \mathrm{~m}^{2}$, $A_{r}=0.05 m^{2}, d=2 m, Z \sim \mathcal{C N}(1,0.2), C=1 \mathrm{bit} / \mathrm{sec} / \mathrm{Hz}$, $\sigma_{i}^{2}=0.001, i \in S_{I}, \sigma_{i}^{2}=0, i \in S_{E}$. We assume the hardware design of [9] is used. According to the measurement data, we set $P_{i n}^{L}=10^{-3} \mu W$ and $P_{i n}^{U}=3200 \mu W$. We impose these constraints in all models to have a fair comparison. We also impose the constraint $\sum_{i} Q_{i} \leq 100 \mathrm{~mW}$ to be consistent with the possible safety and hardware limitations at the transmitter. To plot the actual output power, we utilize a model that uses sinusoidal functions as the basis functions. This model is an overfit to the measurement data and matches

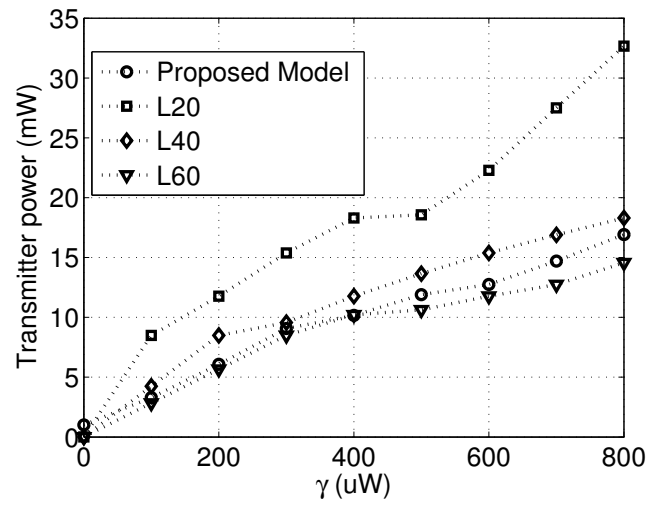

Fig. 4: Transmitter power versus EH constraints

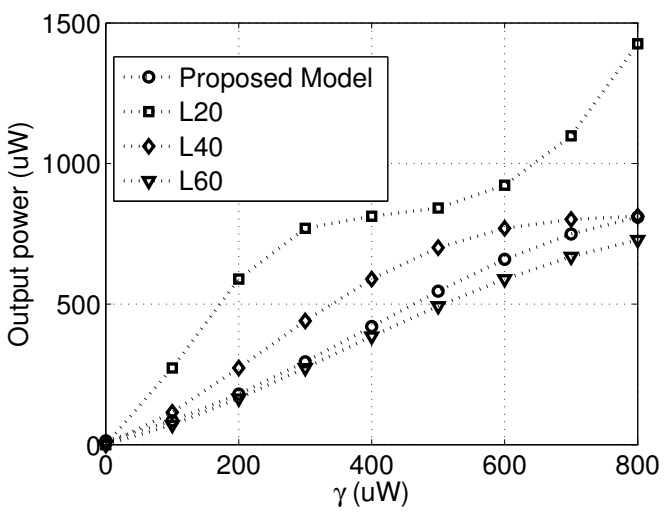

Fig. 5: Output power versus EH constraints

the data very closely; but it is difficult to track analytically. We report average over 100 channel realizations with i.i.d. channel coefficients according to (23). Only the average over feasible scenarios are shown. To compare the results, we also present the performance when (11) is solved using the linear models L20, L40 and L60 of Sec. II. The optimization problems are solved using [20-22].

The transmitter power and the actual power output at the EH receiver for varying EH constraints is shown in Fig. 4 and Fig. 5, respectively. At the the transmitter side, the proposed power for L20 and L40 are higher than the design based on the proposed model, only the transmit power for L60 is under the transmit power of the proposed model. At the energy receiver side, even though L20 and L40 are able to satisfy the EH constraint, the actual power output is high compared to the $\mathrm{EH}$ constraint and this situation can be interpreted as waste of power sources. On the other hand, L60 can not always meet the EH requirements since it is over-optimistic about the power conversion efficiency of the receiver. Hence, we observe that the proposed model provides a reasonable compromise between minimizing transmitter power and satisfying the $\mathrm{EH}$ constraints.

\section{CONCLUSIONS}

We have proposed a quadratic function as a practical RF energy harvesting efficiency model as an alternative to linear models traditionally used SWIPT system design. Comparisons 
between the proposed non-linear model and the linear model as well as comparisons with another non-linear model from the literature are presented. The proposed model is shown to be a good match for the measurement data obtained from practical EH circuity whereas linear models provide considerably poorer match. An attractive property of the proposed model is its convexity properties with respect to input power, which enables ease of analysis and facilitates direct utilization of numerical optimization methods for convex optimization.

Using the proposed model, we have considered a resource allocation problem for a SWIPT OFDMA system. Our results illustrated the importance of using the proposed model compared to typical linear models in the literature. A promising line of future research is the study of the effect of non-linear $\mathrm{EH}$ conversion process in other SWIPT systems. Investigation of the proposed model together with the circuit component models and modeling of other non-linearities in energy harvesting process are also considered as future work.

\section{REFERENCES}

[1] L. Varshney, "Transporting information and energy simultaneously," in IEEE International Symposium on Information Theory (ISIT), 2008, pp. 1612-1616, July 2008.

[2] P. Grover and A. Sahai, "Shannon meets Tesla: Wireless information and power transfer," in IEEE International Symposium on Information Theory (ISIT) 2010, pp. 2363-2367, June 2010.

[3] R. Zhang and C. K. Ho, "MIMO broadcasting for simultaneous wireless information and power transfer," IEEE Trans. Wireless Commun., vol. 12, pp. 1989-2001, May 2013.

[4] A. Nasir, X. Zhou, S. Durrani, and R. Kennedy, "Relaying protocols for wireless energy harvesting and information processing," IEEE Transactions on Wireless Communications, vol. 12, pp. 3622-3636, July 2013.

[5] J. Park and B. Clerckx, "Joint wireless information and energy transfer in a $K$-user MIMO interference channel," IEEE Trans. Wireless Commun., vol. 13, pp. 5781-5796, Oct. 2014.

[6] A. Ozcelikkale and T. M. Duman, "Linear precoder design for simultaneous information and energy transfer over two-user MIMO interference channels," IEEE Trans. Wireless Commun., vol. 14, pp. 5836-5847, Oct 2015.

[7] K. Huang and E. Larsson, "Simultaneous information and power transfer for broadband wireless systems," IEEE Trans. Signal Process., pp. 5972 5986, Dec. 2013.

[8] C. Song, Y. Huang, J. Zhou, S. Yuan, Q. Xu, and P. Carter, "A broadband efficient rectenna array for wireless energy harvesting," in 2015 European Conf. on Antennas and Propagation (EuCAP), pp. 1-5, May 2015.

[9] C. Song, Y. Huang, J. Zhou, J. Zhang, S. Yuan, and P. Carter, "A HighEfficiency Broadband Rectenna for Ambient Wireless Energy Harvesting," IEEE Trans. on Antennas and Propagation, vol. 63, pp. 3486-3495, Aug 2015.

[10] T. Le, K. Mayaram, and T. Fiez, "Efficient far-field radio frequency energy harvesting for passively powered sensor networks," IEEE Journal of Solid-State Circuits, vol. 43, pp. 1287-1302, May 2008.

[11] J. Guo and X. Zhu, "An improved analytical model for RF-DC conversion efficiency in microwave rectifiers," in 2012 IEEE MTT-S International Microwave Symposium Digest, pp. 1-3, June 2012.

[12] B. Clerckx, E. Bayguzina, D. Yates, and P. D. Mitcheson, "Waveform optimization for wireless power transfer with nonlinear energy harvester modeling," in 2015 International Symposium on Wireless Communication Systems (ISWCS), pp. 276-280, 2015.

[13] Y. Huang and B. Clerckx, "Waveform optimization for large-scale multi-antenna multi-sine wireless power transfer," in 2016 IEEE 17th International Workshop on Signal Processing Advances in Wireless Communications (SPAWC), pp. 1-5, July 2016.

[14] B. Clerckx, "Waveform Optimization for SWIPT with Nonlinear Energy Harvester Modeling," arXiv preprint arXiv:1602.01061, 2016.

[15] E. Boshkovska, D. W. K. Ng, N. Zlatanov, and R. Schober, "Practical non-linear energy harvesting model and resource allocation for SWIPT systems," IEEE Comm. Letters, vol. 19, pp. 2082-2085, Dec 2015.
[16] E. Boshkovska, D. W. K. Ng, N. Zlatanov, A. Koelpin, and R. Schober, "Robust Resource Allocation for MIMO Wireless Powered Communication Networks Based on a Non-linear EH Model," arXiv preprint arXiv:1609.03836, 2016

[17] E. Boshkovska, A. Koelpin, D. W. K. Ng, N. Zlatanov, and R. Schober, "Robust Beamforming for SWIPT Systems with Non-linear Energy Harvesting Model," arXiv preprint arXiv:1602.01066, 2016.

[18] E. Boshkovska, R. Morsi, D. W. K. Ng, and R. Schober, "Power Allocation and Scheduling for SWIPT Systems with Non-linear Energy Harvesting Model," arXiv preprint arXiv:1602.00453, 2016.

[19] S. Boyd and L. Vandenberghe, Convex Optimization. Cambridge University Press, 2004

[20] J. F. Sturm, "Using SeDuMi 1.02, a Matlab toolbox for optimization over symmetric cones," Optimization Methods and Software, vol. 11, no. $1-4$, pp. $625-653,1999$.

[21] R. H. Tütüncü, K. C. Toh, and M. J. Todd, "Solving semidefinitequadratic-linear programs using SDPT3," Mathematical Programming, vol. 95 , no. 2, pp. 189-217, 2003.

[22] CVX Research Inc., "CVX: Matlab software for disciplined convex programming 2.0." http://cvxr.com/cvx, 2012.

[23] B. Gupta and I. Guttman, Statistics and Probability with Applications for Engineers and Scientists. Wiley, 2013.

[24] A. W. Marshall and I. Olkin, Inequalities: Theory of Majorization and its Applications. Academic Press, 1979.

[25] W. C. Brown and E. E. Eves, "Beamed microwave power transmission and its application to space," IEEE Trans. on Microwave Theory and Techniques, vol. 40, pp. 1239-1250, Jun 1992. 